\title{
Residency Support During a Natural Disaster: Hurricane Ida
}

Tiffany Chang, MD, Elizabeth W. Kelly, MD, Chi Dola, MD

\section{Abstract}

Author affiliations are listed at the end of this article.

Correspondence to:

Chi Dola, MD

Tulane University

1430 Tulane Avenue

Box 8611

New Orleans, LA 70112

(cdola@tulane.edu) Hurricane Ida on the 16th anniversary of Hurricane Katrina. Our medical workforce faced challenges caring for patients in a facility without potable water and powered by a generator. With the support of corporate entities and our academic institution, however, these barriers were quickly overcome and thus highlights the importance of collective resilience in the face of a natural disaster.

\section{Keywords}

natural disasters; cyclonic storms; internship and residency; New Orleans; multi-institutional systems

\section{To the Editor:}

Hurricane Ida made landfall as a dangerous Category 4 hurricane along the Gulf Coast of Louisiana on August 29, 2021, the 16th anniversary of Hurricane Katrina. It ravaged our communities and caused significant damage to fundamental infrastructure, including power, municipal water, food supply, housing and transportation. These impacts have been particularly devastating in a region vulnerable to natural disasters and healthcare disparities. The hurricane disrupted our healthcare system, limiting access to basic medical care and clinical services.

In anticipation of and preparation for Hurricane Ida, the Tulane School of Medicine Graduate Medical Education office, in collaboration with affiliated training hospitals, deployed "Code Gray" teams to provide emergency services at our clinical facilities. As obstetricians and gynecologists, we focused on the needs of pregnant women and their infants, along with emergency gynecological services at Tulane-Lakeside Hospital, which serves the greater New Orleans metropolitan area. In the aftermath of Hur- ricane Ida, our primary obstetrics facility was severely impacted. Although electricity was maintained with generators, the loss of municipal water supply affected our capability to provide adequate obstetrical care and services. However, these barriers were quickly overcome with the reparations and generous support from corporate entities within HCA Healthcare. (Figure 1) Specifically, the operating HCA Healthcare facility with obstetrical services, Lakeview Regional Medical Center, located approximately 30 miles away, was identified, and women and infants were safely evacuated by ambulance or helicopter in a timely and orderly fashion.

Our workforce, including attending and resident physicians, registered nurses and other ancillary staff, was also relocated to the sister HCA Healthcare facility to continue caring for our patients. Although this transition was facilitated by a warm welcome by the new facility, the aftermath of Ida had proved more difficult than expected for remaining individuals stranded with limited or inadequate access to basic necessities. As physicians, the feasibility

\section{HCA 许 Healthcare}

www.hcahealthcarejournal.com

(C) 2021 HCA Physician Services, Inc. d/b/a Emerald Medical Education
HCA Healthcare Journal of Medicine 


\author{
Hurricane Ida \\ Hurricane Ida \\ makes landfall in \\ southeast \\ Lousiana
}

\author{
Patient Transfers \\ Patients trans- \\ ported to nearby \\ hospital, Lakeview \\ Regional Medical \\ Center
}

\author{
Food and Shelter \\ Food and shelter \\ provided at no \\ cost to health care \\ teams
}

Power Restoration

Power is restored to hospitals in the greater

New Orleans area

Additional Housing

Hotel accomodations

available for team

members

Laundry services available at affected

HCA Healthcare facilities at no cost

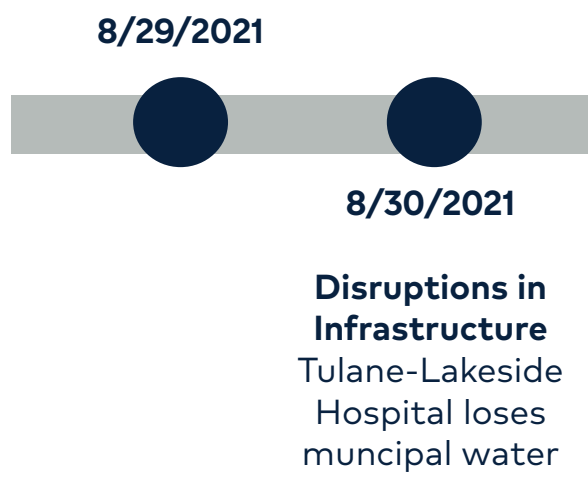

$8 / 31 / 2021$

9/2/2021

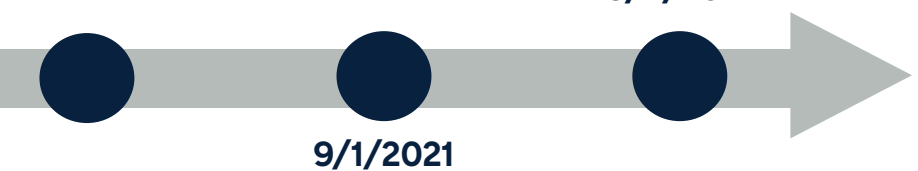

Transportation and

Basic Needs

Gasoline (available

onsite at all affect-

ed HCA Healthcare

facilities) and hotel

accomodations

provided at no cost

Figure 1. A timeline shows events after Hurricane Ida made landfall.

of providing care was not only confounded by these disruptions but also contingent upon the initial emergency response, including mobilization of crucial resources. In a matter of hours, HCA Healthcare quickly compounded available resources to address our essential needs. Food, water and shelter were immediately allocated for our team within the nearby hospital. Hotel accommodations were also provided to team members with homes that sustained damage from the storm or were devoid of water and electricity. This rapid, coordinated emergency response ensured the security of basic human needs and thus allowed our workforce to focus on medical care.

Stable access to water, shelter and sanitation was then followed by provisions to ensure the availability of other necessities, including transportation. Fuel supply, already depleted from emergency preparations, was further disrupted by the impacts of Hurricane Ida, which halted the supply chain for critical resources, including gasoline. The fuel shortage hindered our workforce: eager to help, but limited by access to transportation. Within 24 hours, multiple gasoline tank trucks arrived at each affected HCA Healthcare facility and provided gasoline, at no cost, for transportation, as well as portable home generators. Mobile laundry trailers were also deployed to our affected facilities, providing emergency team members with convenient onsite access to laundry appliances and sanitation.

Tulane School of Medicine's medical student and faculty volunteers provided additional support by delivering non-perishable food items and toiletries. Furthermore, designated staff members worked diligently to identify additional shelter at local hotels for individuals whose homes had been devastated by the hurricane or made uninhabitable. The medical school continued to allocate free hotel rooms 
for our team members even after power and water were restored, in order to accommodate those unable to travel the greater distance home at the end of a work shift.

With the remaining workforce, in addition to evacuees displaced to closer living quarters, many faced an increased risk of exposure to and transmission of infectious diseases. The ongoing COVID-19 pandemic, now complicated by a disrupted medical care system, posed a serious public health challenge. However, rapid SARS-CoV-2 testing sites remained operational due to anticipation by and preparedness of the Tulane School of Medicine. Furthermore, as these catastrophic conditions can be devastating on emotional, social and physical wellbeing, the medical school ensured reliable access to resources and support. Collectively, the rapid response and strategic planning by Tulane School of Medicine and HCA Healthcare to address our basic needs proved not only highly effective but also a silver lining in the devastation imparted by Hurricane Ida.

Our experiences before, during and after Hurricane Ida serve as a reminder of the health care disturbances that may occur during pregnancy. Most importantly, the recent events illustrate the crucial support of medical schools and corporate entities in disaster preparedness to rapidly mobilize resources and thereby preclude potential barriers to health care delivery and team morale. Our healthcare workers share the utmost gratitude for our institutions that rapidly mobilized an effective emergency response, compounded essential resources and thus promoted our delivery of medical care in the aftermath of a destructive natural disaster. Collectively, our experiences illuminate the exemplary collaboration and commitment of our dedicated support staff to manifest "resident wellness," a state we now re-envision in the aftermath of Hurricane Ida. We hope that sharing our experiences and the lessons learned from Hurricane Ida may ultimately foster the development and refinement of future hurricane and emergency response plans at other academic institutions.

\section{Conflicts of Interest}

The authors declare they have no conflicts of interest.
The authors are affiliated with Tulane University Medical Center, a hospital affiliated with the journal's publisher.

This research was supported (in whole or in part) by HCA Healthcare and/or an HCA Healthcare affiliated entity. The views expressed in this publication represent those of the author(s) and do not necessarily represent the official views of HCA Healthcare or any of its affiliated entities.

\section{Author Affiliations}

1. Tulane University School of Medicine, New Orleans, LA 